\title{
Sowing density of herbage and seed production from two Jack bean (Canavalia
} ensiformis (L.) DC) cultivars

${ }^{1}$ Jolaosho, A. O., ${ }^{2}$ Akinola, J. O., ${ }^{1}$ Okukenu, O. A., ${ }^{2}$ Binuomote, R. T. and ${ }^{3}$ Odedire, J. A

${ }^{I}$ Department of Pasture and Range Management, College of Animal Science, Federal University of Agriculture, Abeokuta, Ogun State, Nigeria.

${ }^{2}$ Department of Animal Production and Health, Ladoke Akintola University of Technology, Ogbomoso, Oyo State, Nigeria

${ }^{3}$ Department of Animal Science, ObafemiAwolowo University, Ile-Ife, Osun State, Nigeria

Abstract Corresponding author:jolaoshoao@funaab.edu.ng: +234805550447

Grain legumes are important foodstuffs in most of the tropical and subtropical countries, where they are second only to cereals as a source of protein. The grain legumes are rich in protein and can be considered as a natural supplement to cereals. This experiment was carried out to determine the effects of seven sowing densities -10.0, 13.3, 17.8, 20.0, 26.7, 40.0 and $80.0 \times 10^{3}$ plants/ ha on leaf dry matter yield (LDMY), stem dry matter yield (SDMY), seed yield (SY) components, leaf crude protein content (LCP) and seed crude protein content (SCP) of two Jack bean (Canavalia ensiformis) cultivars. The trial, sited $4 \mathrm{~km}$ from the Osun College of Education, Ilesa, Osun State, south western Nigeria, was conducted during the 2011 and 2012 planting seasons. In both experimental years, the brown-seeded cultivar had a significantly higher LDMY, SDMY and SCP content than the white-seeded cultivar. While the cultivars could not be differentiated on LCP content basis, the white-seeded cultivar recorded significantly higher SY, seed/ pod ratio and 100 -seed weight values. For both years, the highest LDMY was produced by the brown-and white-seeded cultivars at the sowing densities of 20.0 and $26.7 \times 10^{3}$ plants/ ha, respectively, compared with the highest SY that was produced at $17.8 \times 10^{3}$ plants /ha regardless of cultivar. The study indicated the potential of the brown-and white-seeded cultivars for herbage and seed yields, respectively. Keywords: Sowing density, herbage, seed, Jack bean

\section{Densité de semis de la production d'herbe et de graines de deux cultivars de haricot gris (Canavalia ensiformis (L.) DC)}

\section{Résumé}

$\overline{L e s}$ légumineuses à grains sont des denrées alimentaires importantes dans la plupart des pays tropicaux et subtropicaux, où elles viennent juste après les céréales comme source de protéines. Les légumineuses à grains sont riches en protéines et peuvent être considérées comme un complément naturel aux céréales. Cette expérience a été réalisée pour déterminer les effets de sept densités de semis -10,0, 13,3, 17,8, 20,0, 26,7, 40,0 et 80,0 x 103 plants/ha sur le rendement en matière sèche des feuilles (RMSF), le rendement en matière sèche de la tige (RMST), les graines les composants du rendement (CR), la teneur en protéines brutes des feuilles $(P B F)$ et la teneur en protéines brutes des graines (SCP) de deux cultivars de haricot gris (Canavalia ensiformis). L'essai, situé à $4 \mathrm{~km}$ de l'Osun College of Education, à Ilesa, dans l'État d'Osun, au sud-ouest du Nigéria, a été mené pendant les saisons de plantation 2011 et 2012. Au cours des deux années expérimentales, le cultivar à graines brunes avait une teneur en RMSF, RMST et SCP significativement plus élevée que le cultivar à graines blanches. Alors que les cultivars n'ont pas pu être différenciés sur la base de la teneur en PBF, 
le cultivar à graines blanches a enregistré des valeurs de $C R$, de rapport graine/gousse et de poids de 100 graines significativement plus élevées. Pour les deux années, le RMSF le plus élevé a été produit par les cultivars à graines brunes et blanches à des densités de semis de 20,0 et 26,7 x 103 plants/ha, respectivement, par rapport au SY le plus élevé qui a été produit à 17,8 $\times 103$ plants/ha indépendamment de cultivar. L'étude a indiqué le potentiel des cultivars à graines brunes et blanches pour les rendements en herbage et en graines, respectivement.

Mots clés : Densité de semis, herbage, graine, haricot Jack

\section{Introduction}

Legumes are rich in protein and their chemical composition varies depending on variety, species and region. The protein content of legumes is twice or triple that of cereals depending on the type of the legume. The protein of legumes though adequate in essential amino acid lysine is however deficient in sulphur containing amino acids, methionine and cystine. Legumes, however, forms good supplements for cereals which are lacking in essential amino acid lysine. Improved nutritional quality can therefore be achieved by combining legumes with cereals. Most legumes are low sources of fat with the exception of soybean and groundnut. Legumes are also good sources of different minerals such as calcium and phosphorus (Liu, 1997). The bioavailability of these minerals can be improved through processing. Legumes contain antinutritional factors such as lectins, saponin, haemagglutin, protease inhibitor, oxalate, goitrogen, phytates, trypsin inhibitor and tannin. These compounds reduce protein digestibility and availability and some antinutritional factors in legumes have been reported to have health benefits. Tannin, a polyphenolic compound is reported to possess anti-oxidative activity (Amarowicz and Pegg, 2008). Pulses are grown as food crops for their protein content and the oilseeds like soybean and groundnut are grown as commercial crops for their oil contents. The oil is extracted either by heat and pressure (mechanically) and/or by solvent extraction. The residual cake/meal, rich in protein, is used mainly for animal and aqua feed. However, where appropriate technology and hygiene control is maintained, the cake/meal can be made edible for human consumption.Jack bean (Canavaliaensiformis), is a perennial or annual herb (Bogdan, 1977), is a stout, deep rooted, drought tolerant, creeping and twining/climbing legume. Although, native to tropical African, South and Central America, it is now naturalized and cultivated worldwide (USDA, 2009). It is much better adapted than most other pulses to a wide range of rainfall $(800-2000 \mathrm{~mm})$ , altitude $(0-1800 \mathrm{~m})$, soil texture and soil fertility conditions. In addition, the Jack bean strives well on nutrient depleted, highly leached, acidic ( $\mathrm{pH} 4.3$ - 6.8), waterlogged and saline lowland tropical soils (NAS/ NRC, 1979). It is recognized as a commercial crop for being relevant, not only in human (Udedibie and Carlini, 1998; Adebowale and Lawal, 2004) and livestock nutrition (Heuze and Tran, 2015; Akingbadeet al., 2007), although the seeds and foliage contains several anti-nutritional factors such as concanalin A, canavanine and canatoxin which can lead to fever, nasal discharge, lameness and prostration in cattle (Chees et al., 1992) or death (FAO 2009). It can also be used in green manuring and cover cropping (Brazill, 1987; Ferreira et al., 2013), soil biofumigation (Fischler and wortmann, 1999; Morris and Walker, 2002; Arimet al, 2006), aquaculture (Jimohet al., 2010) and ethnobotany (Olowokudejoet al., 2008) in addition to phytoremediation of lead in soils (Pereira et 


\section{Jolaosho, Akinola, Okukenu, Binuomote and Odedire}

al., 2010) globally and especially in the tropics. The objective of the study was to investigate the leaf, stem and seed yield and quality reactions to an array of plant population densities of two Jack been cultivars that differ in seed shape, size and testacolour.

\section{Materials and methods}

\section{Location, land preparation and cultivar}

The experiment was sited about $4 \mathrm{~km}$ from the Osun College of Education, Ilesa $\left(7^{0} 36^{\circ} \mathrm{N}, 4^{0} 42 \mathrm{E}, 396 \mathrm{~m}\right)$ in the Forest Zone of south- western Nigeria Meteorological observations at the ObafemiAwolowo University, Ile- Ife $\left(7^{0} 28^{\prime} \mathrm{N}, 4^{0} 38^{\prime}\right.$ E, $244 \mathrm{~m}$ asl) $25 \mathrm{~km}$ west of Ilesa, during the trial as presented in Table 1. The site was cropped with maize without any fertilizer application in 2010. Before planting in 2011, the top soil $(0-15 \mathrm{~cm})$ had the following physico chemical properties$72.1 \%$ sand, $10.5 \%$ silt, $17.4 \%$ clay, $\mathrm{pH}$ ( $1: 1$, soil : water) $5.9,2.35 \%$ organic carbon, $0.13 \%$ total $\mathrm{N}$ and $6.83 \mathrm{ppm}$ available $\mathrm{P}$ (Bray 1).Seed bed preparation consisted of ploughing twice, and harrowing with addition of single superphosphate application by raking in at the rate equivalent to $20 \mathrm{kgP} / \mathrm{ha}$. A white-seeded Jack bean cultivar from the International Institute of Tropical Agriculture and a brown -seeded cultivar from behind an Ilesa motor mechanical workshop were examined. The former was egg-shaped, $20 \mathrm{~mm}$ long, $14 \mathrm{~mm}$ wide and $12.5 \mathrm{~mm}$ thick while the latter, somewhat flat, measured $27 \mathrm{~mm}$ long, $16 \mathrm{~mm}$ wide and $8.5 \mathrm{~mm}$ thick. Both had been grown annually and harvested for seed since 2006.

\section{Experimental design and management}

A randomized complete block design with three replicates was used. Two healthy seeds were hand sown per hill at $2 \mathrm{~cm}$ depths in $6 \mathrm{~m} \mathrm{x} 6 \mathrm{~m}$ plots on $1^{\text {st }}$ August, 2011 and $30^{\text {th }} \mathrm{July}, 2012$. Seedlings were thinned to one per hill 21 days after sowing at each of the $100 \times 100,100 \times 75,75 \times 75,100 \times 50$,
$75 \times 50,50 \times 50$ and $50 \times 25 \mathrm{~cm}$ spacing that provided 10.0, 13.3, 17.8, 20.0, 26.7, 40.0 and $80.0 \times 10^{3}$ plants/ ha, respectively. Plots and replicates were demarcated by $1.5 \mathrm{~m}$ borders. Weed control was manual, and applied once after five weeks of sowing.

\section{Yield determination}

Herbage yield was evaluated at 50\% flowering on 7th November, 2011 and 5th November, 2012 while seed yield was determined when all pods had desiccated on 30th January, 2012 and $26^{\text {th }}$ January, 2013. Two $2 \mathrm{~m} \times 2 \mathrm{~m}$ quadrats per plot were sampled for either herbage or seed. Plants cut at the cotyledonary node were weighed and $500-530 \mathrm{~g}$ sub- samples taken and separated into the leaf (petiole + leaf lamina) and stem (main stem, branches and inflorescences) fractions. These were ovendried at $80^{\circ} \mathrm{C}$ for 48 hours. From the handpicked and weighed pods, 30 were randomly taken for empty pod and seed measurements and the rest threshed for seed collection. Empty pods and seeds were weighed, oven -dried as applied to the herbage and re-weighed to calculate 100seed weight, dry seed weight to dry empty pod weight ratio and seed yield / ha.

\section{Chemical and statistical analyses}

The oven-dried leaf and seed fractions were milled and analyzed for crude protein concentration according to AOAC (1995). Data collected (herbage yield, seed yield components, herbage and seed crude protein contents) were analyzed on a yearly basis according to the techniques outlined by Gomez and Gomez (1984) using the General Linear Model (GLM) Procedure of the Statistical Analysis System (SAS, 1988) package.

\section{Results and discussion}

Rainfall during the experiment (August January) was similar for both years, $661.7 \mathrm{~mm}$ (2011) and 665.8mm (2012) but the distribution was unidentical(Table 1). Lower temperature and relative humidity 
characterized the harmattan period of pod development (December) in 2011.

Overall, the brown - seeded cultivar produced higher leaf and stem dry matter yields but lower seed yield than the white -seeded cultivar in 2011 (Table 2). The white seeded cultivar was greater in seed / pod ratio and 100- seed weight values. Both cultivars recorded similar leaf crude protein contents but the white-seeded cultivar had significantly lower seed crude protein content. Leaf dry matter yield maximized at sowing densities of $20.0 \times 10^{3}$ and $26.7 \times 10^{3}$ plants / ha for the brown-and white-seeded cultivars, respectively, but for both cultivars, the highest stem dry matter yield was observed at $40 \times 10^{3}$ plants/ ha (Table $3)$. Whereas the seed/ pod ratio tended to increase with increasing sowing density, 100-seed weight decreased, regardless of cultivar. The optimum population density for seed yield was $17.8 \times 10^{3}$ plants / ha. Sowing density had no significant influence on the leaf crude protein content while the seed crude protein content appeared to have declined with denser sowing. The result obtained in 2012 followed the general trend of those observed in 2011 in respect of cultivar differences (Table 4). However, the plants produced slightly higher figures in the second than the first year especially in terms of leaf and stem dry matter yields, leaf crude protein content and 100- seed weight for the brown -seeded cultivar and seed yield, seed/ pod ratio, 100-seed weight and seed crude protein content for the white -seeded cultivar. The brown -seeded cultivar produced higher stem dry matter yield at $20 \times 10^{3}$ plants/ ha than at lower or higher densities while, for the white seeded cultivar, stem dry matter yield apparently rose with increasing sowing density (Table 5). The levels of dry matter yield recorded in this study supersede those of $2064-3698 \mathrm{~kg} / \mathrm{ha}$ and $772-1661 \mathrm{~kg} / \mathrm{ha}$ for the leaf and stem yields, respectively, reported for the white- seeded cultivar grown at $10-26.7 \times 10^{3}$ plants/ha at Ogbomoso in the derived savanna zone, a drier Nigerian environment (Binuomoteet al., 2009). The brown-seeded cultivar, with its superior leaf yield and high leaf crude protein content can be recommended to supplement low quality hay or dry season grazing. On the other hand, the higher seed yielding white - seeded cultivar should be suitable for concentrate feed production.

Table 1: Monthly rainfall $(\mathrm{mm})$, temperature $\left({ }^{\circ} \mathrm{C}\right)$ and relative humidity $(\%)$ at the ObafemiAwolowo University, Ile-Ife during the experiment

\begin{tabular}{|c|c|c|c|c|c|c|c|c|c|c|c|c|}
\hline \multirow[t]{3}{*}{ Month } & \multicolumn{3}{|c|}{ Rainfall } & \multicolumn{6}{|c|}{ Temperature } & \multicolumn{3}{|c|}{ Relative humidity } \\
\hline & \multirow[t]{2}{*}{2011} & \multirow[t]{2}{*}{2012} & \multirow[t]{2}{*}{2013} & \multicolumn{2}{|c|}{2011} & \multicolumn{2}{|c|}{2012} & \multicolumn{2}{|c|}{2013} & \multirow[t]{2}{*}{2011} & \multirow[t]{2}{*}{2012} & \multirow[t]{2}{*}{2013} \\
\hline & & & & $\operatorname{Max}$ & Min & $\operatorname{Max}$ & Min & Max & Min & & & \\
\hline Jan & 6.4 & 0.0 & 0.0 & 34.4 & 15.5 & 35.1 & 15.2 & 38.3 & 13.7 & 54.5 & 57.9 & 60.7 \\
\hline Feb & 62.7 & 98.8 & - & 35.3 & 20.8 & 34.8 & 20.3 & - & - & 71.3 & 73.9 & - \\
\hline Mar & 74.9 & 23.4 & - & 35.7 & 21.4 & 36.4 & 20.0 & - & - & 75.3 & 70.3 & - \\
\hline Apr & 58.9 & 60.5 & - & 35.3 & 20.4 & 35.3 & 21.1 & - & - & 75.5 & 76.8 & - \\
\hline May & 177.8 & 0.0 & - & 33.9 & 20.9 & 34.4 & 19.5 & - & - & 79.8 & 80.1 & - \\
\hline June & 204.2 & 206.5 & - & 32.3 & 20.9 & 32.3 & 20.1 & - & - & 79.5 & 83.1 & - \\
\hline July & 205.0 & 188.7 & - & 30.8 & 20.1 & 29.8 & 20.0 & - & - & 86.6 & 86.9 & - \\
\hline Aug & 127.0 & 49.8 & - & 29.9 & 20.1 & 29.5 & 20.0 & - & - & 86.3 & 87.8 & - \\
\hline Sep & 274.8 & 227.6 & - & 31.7 & 19.9 & 31.1 & 19.8 & - & - & 85.3 & 84.2 & - \\
\hline Oct & 196.4 & 249.7 & - & 31.8 & 20.0 & 31.8 & 20.2 & - & - & 84.4 & 83.9 & - \\
\hline Nov & 63.5 & 129.6 & - & 33.7 & 20.1 & 33.7 & 20.5 & - & - & 77.1 & 80.3 & - \\
\hline Dec & 0.0 & 9.1 & - & 35.1 & 15.0 & 34.9 & 17.2 & - & - & 57.9 & 71.9 & - \\
\hline Total/mean & 1451.6 & 1243.7 & - & 33.3 & 19.6 & 33.3 & 22.0 & & & 76.1 & 78.1 & \\
\hline
\end{tabular}

* Interestingly, rainfall during the experiment (Aug - Dec) is $661.7 \mathrm{~mm}$ in 2011 and $665.8 \mathrm{~mm}$ in 2012 
Table 2: Main effects of cu ltivars and sowing densities on yield parameters and crude protein (CP) contents of leaves and seeds of Canavaliaensiformis in 2011

\begin{tabular}{llllllll}
\hline \multicolumn{7}{c}{ Parameters } \\
\hline Cultivars & $\begin{array}{l}\text { LDMY } \\
(\mathrm{kg} / \mathrm{ha})\end{array}$ & $\begin{array}{l}\text { SDMY } \\
(\mathrm{kg} / \mathrm{ha})\end{array}$ & $\begin{array}{l}\text { LCP } \\
(\% \mathrm{DM})\end{array}$ & $\begin{array}{l}\text { SY } \\
(\mathrm{kg} / \mathrm{ha})\end{array}$ & S:P & $\begin{array}{l}\text { 100-seed } \\
\text { weight }(\mathrm{g})\end{array}$ & $\begin{array}{l}\text { SCP } \\
(\% \mathrm{DM})\end{array}$ \\
\hline Black & $4380.80^{\mathrm{a}}$ & $8399.30^{\mathrm{a}}$ & 23.98 & $2632.10^{\mathrm{b}}$ & $1.25^{\mathrm{b}}$ & $459.75^{\mathrm{b}}$ & $34.93^{\mathrm{a}}$ \\
White & $3876.00^{\mathrm{b}}$ & $7382.5^{\mathrm{b}}$ & 23.40 & $2891.80^{\mathrm{a}}$ & $1.57^{\mathrm{a}}$ & $529.64^{\mathrm{a}}$ & $33.32^{\mathrm{b}}$ \\
SEM & 137.35 & 449.64 & 0.34 & 131.44 & 0.02 & 9.38 & 0.42 \\
Sowing density $\left(\mathbf{X 1 0}^{3}\right.$ plants/ha) & & & & & \\
10 & $3749.20^{\mathrm{cd}}$ & $4893.50^{\mathrm{e}}$ & 24.67 & $2497.00^{\mathrm{cd}}$ & $1.34^{\mathrm{c}}$ & $551.97^{\mathrm{a}}$ & $35.62^{\mathrm{a}}$ \\
13.3 & $4156.20^{\mathrm{bc}}$ & $5861.30^{\mathrm{d}}$ & 23.98 & $3192.20^{\mathrm{b}}$ & $1.34^{\mathrm{c}}$ & $544.17^{\mathrm{a}}$ & $34.90^{\mathrm{ab}}$ \\
17.8 & $4424.80^{\mathrm{ab}}$ & $6882.30^{\mathrm{c}}$ & 24.70 & $3601.70^{\mathrm{a}}$ & $1.37^{\mathrm{c}}$ & $514.62^{\mathrm{b}}$ & $34.33^{\mathrm{abc}}$ \\
20 & $4817.70^{\mathrm{a}}$ & $8518.20^{\mathrm{b}}$ & 22.86 & $2951.50^{\mathrm{b}}$ & $1.40^{\mathrm{bc}}$ & $483.67^{\mathrm{c}}$ & $34.53^{\mathrm{abc}}$ \\
26.7 & $4507.20^{\mathrm{ab}}$ & $9514.20^{\mathrm{a}}$ & 23.25 & $2859.30^{\mathrm{bc}}$ & $1.44^{\mathrm{b}}$ & $466.23^{\mathrm{cd}}$ & $34.35^{\mathrm{abc}}$ \\
40 & $3890.70^{\mathrm{c}}$ & $9866.20^{\mathrm{a}}$ & 22.85 & $2275.20^{\mathrm{de}}$ & $1.46^{\mathrm{ab}}$ & $462.93^{\mathrm{d}}$ & $32.87^{\mathrm{bc}}$ \\
80 & $3353.00^{\mathrm{d}}$ & $9700.70^{\mathrm{a}}$ & 23.48 & $1957.00^{\mathrm{e}}$ & $1.51^{\mathrm{a}}$ & $439.30^{\mathrm{e}}$ & $32.28^{\mathrm{c}}$ \\
SEM & 199.95 & 376.20 & 0.59 & 131.03 & 0.07 & 16.63 & 0.77 \\
\hline
\end{tabular}

a, b, c, d, e :Means in same column with different superscripts are significantly $(\mathrm{p}<0.05)$ different

SEM=Standard Error of Mean, LDMY =Leaf dry matter yield, SDMY $=$ Stem dry matter yield

$\mathrm{LCP}=$ Leaf CP content, $\mathrm{SY}=$ Seed yield, $\mathrm{S}: \mathrm{P}=$ Seed:Pod ratio, $\mathrm{SCP}=$ Seed CP content

Table 3: Interaction effects of cultivar and sowing density differences on yield paremeters and crude protein (CP) contents of leaves and seeds of Canavaliaensiformis in 2011

\begin{tabular}{|c|c|c|c|c|c|c|c|c|}
\hline \multicolumn{9}{|c|}{ Parameters } \\
\hline Cultivars & $\begin{array}{l}\text { Sowing } \\
\text { density }\end{array}$ & $\begin{array}{l}\text { LDMY } \\
(\mathrm{kg} / \mathrm{ha})\end{array}$ & $\begin{array}{l}\text { SDMY } \\
(\mathrm{kg} / \mathrm{ha})\end{array}$ & $\begin{array}{l}\text { LCP } \\
(\% \mathrm{DM})\end{array}$ & $\begin{array}{l}\text { SY } \\
(\mathrm{Kg} / \mathrm{ha})\end{array}$ & S:P & $\begin{array}{l}100 \text {-seed } \\
\text { weight }(\mathrm{g})\end{array}$ & $\begin{array}{l}\text { SCP } \\
(\% \mathrm{DM})\end{array}$ \\
\hline \multirow[t]{7}{*}{ Black } & 10 & $3991.00^{\text {cdef }}$ & $4824.30^{\mathrm{e}}$ & $25.23^{\mathrm{a}}$ & $2381.30^{\text {cde }}$ & $1.20^{\mathrm{g}}$ & $508.43^{\mathrm{c}}$ & $36.43^{\mathrm{a}}$ \\
\hline & 13.3 & $4367.30^{\mathrm{bcd}}$ & $6361.30^{\mathrm{cd}}$ & $24.43^{\mathrm{a}}$ & $2956.30^{\mathrm{bc}}$ & $1.23^{\mathrm{fg}}$ & $496.17^{\mathrm{cd}}$ & $34.57^{\mathrm{abcd}}$ \\
\hline & 17.8 & $5019.30^{\mathrm{ab}}$ & $7948.30^{b}$ & $24.77^{\mathrm{a}}$ & $3361.30^{\mathrm{ab}}$ & $1.19^{\mathrm{g}}$ & $474.33^{\text {de }}$ & $35.33^{\mathrm{abc}}$ \\
\hline & 20 & $5601.70^{\mathrm{a}}$ & $9745.70^{\mathrm{a}}$ & $22.83^{\mathrm{a}}$ & $2895.70^{b c}$ & $1.24^{\mathrm{fg}}$ & $458.00^{\mathrm{ef}}$ & $36.20^{\mathrm{b}}$ \\
\hline & 26.7 & $4512.00^{b c}$ & $9677.30^{\mathrm{a}}$ & $24.03^{\mathrm{a}}$ & $2852.30^{b c}$ & $1.27^{\mathrm{fg}}$ & $438.53^{\mathrm{f}}$ & $34.94^{\mathrm{abc}}$ \\
\hline & 40 & $3774.30^{\operatorname{def}}$ & $10203.00^{\mathrm{a}}$ & $22.90^{\mathrm{a}}$ & $2141.70^{\text {de }}$ & $1.27^{\mathrm{fg}}$ & $431.97^{\mathrm{fg}}$ & $33.70^{\mathrm{abcd}}$ \\
\hline & 80 & $3400.00^{\text {ef }}$ & $10035.30^{\mathrm{a}}$ & $23.63^{\mathrm{a}}$ & $1836.30^{\mathrm{e}}$ & $1.32^{\mathrm{f}}$ & $410.83^{\mathrm{g}}$ & $33.33^{\text {abcd }}$ \\
\hline \multirow[t]{7}{*}{ White } & 10 & $3507.30^{\text {ef }}$ & $4962.70^{\mathrm{e}}$ & $24.10^{\mathrm{a}}$ & $2612.70^{\text {cd }}$ & $1.48 \mathrm{~d}^{\mathrm{e}}$ & $595.50^{\mathrm{a}}$ & $34.80^{\mathrm{abc}}$ \\
\hline & 13.3 & $3945.00^{\text {cdef }}$ & $5361.30^{\text {de }}$ & $23.53^{\mathrm{a}}$ & $3428.00^{\mathrm{b}}$ & $1.44^{e}$ & $592.17^{\mathrm{a}}$ & $35.23^{\mathrm{abc}}$ \\
\hline & 17.8 & $3830.30^{\text {cdef }}$ & $5816.30^{\text {de }}$ & $24.63^{\mathrm{a}}$ & $3842.00^{\mathrm{a}}$ & $1.54^{\mathrm{cd}}$ & $554.90^{\mathrm{b}}$ & $33.33^{\text {abcd }}$ \\
\hline & 20 & $4033.70^{\text {cde }}$ & $7290.70^{b c}$ & $22.90^{\mathrm{a}}$ & $3007.30^{\text {bc }}$ & $1.55^{\mathrm{bcd}}$ & $509.33^{c}$ & $32.87^{\mathrm{bcd}}$ \\
\hline & 26.7 & $4502.30^{\mathrm{bc}}$ & $9351.00^{\mathrm{a}}$ & $22.47^{\mathrm{a}}$ & $2866.30^{\mathrm{bc}}$ & $1.61^{\mathrm{abc}}$ & $493.93^{\text {cd }}$ & $33.77^{\mathrm{abcd}}$ \\
\hline & 40 & $4007.00^{\text {cdef }}$ & $9529.30^{\mathrm{a}}$ & $22.80^{\mathrm{a}}$ & $2408.70^{\text {cde }}$ & $1.64^{\mathrm{ab}}$ & $493.90^{\text {cd }}$ & $32.03^{\text {cd }}$ \\
\hline & 80 & $3306.00^{\mathrm{f}}$ & $9366.00^{\mathrm{a}}$ & $23.33^{\mathrm{a}}$ & $2077.70^{\text {de }}$ & $1.70^{\mathrm{a}}$ & $467.77^{\mathrm{de}}$ & $31.23^{\mathrm{d}}$ \\
\hline SEM & & 106.24 & 324.05 & 0.24 & 94.36 & 0.03 & 8.61 & 0.32 \\
\hline
\end{tabular}


Table 4: Main effects of cultivars and sowing densities on yield $p$ arameters and crude protein (CP) contents of leaves and seeds of Canavaliaensiformis in 2012

\begin{tabular}{|c|c|c|c|c|c|c|c|}
\hline \multirow[b]{2}{*}{ Cultivars } & \multirow[b]{2}{*}{$\begin{array}{l}\text { LDMY } \\
\text { (kg/ha) }\end{array}$} & \multirow[b]{2}{*}{$\begin{array}{l}\text { SDMY } \\
(\mathrm{kg} / \mathrm{ha})\end{array}$} & \multicolumn{5}{|c|}{ Parameters } \\
\hline & & & $\begin{array}{l}\mathrm{LCP} \\
(\% \mathrm{DM})\end{array}$ & $\begin{array}{l}\mathrm{SY} \\
(\mathrm{Kg} / \mathrm{ha})\end{array}$ & S:P & $\begin{array}{l}100 \text {-seed } \\
\text { weight }(\mathrm{g})\end{array}$ & $\begin{array}{l}\text { SCP } \\
(\% \mathrm{DM})\end{array}$ \\
\hline Black & $5132.90^{\mathrm{a}}$ & $8550.70^{\mathrm{a}}$ & 24.67 & $2632.20^{\mathrm{b}}$ & $1.25^{\mathrm{b}}$ & $483.81^{\mathrm{b}}$ & $34.88^{\mathrm{a}}$ \\
\hline White & $3838.00^{\mathrm{b}}$ & $6834.00^{\mathrm{b}}$ & 23.70 & $3340.90^{\mathrm{a}}$ & $1.58^{\mathrm{a}}$ & $569.79^{\mathrm{a}}$ & $33.63^{\mathrm{b}}$ \\
\hline SEM & 146.16 & 479.33 & 0.37 & 141.69 & 0.02 & 8.16 & 0.41 \\
\hline \multicolumn{8}{|c|}{ Sowing density (X10 3 plants $/ \mathrm{ha})$} \\
\hline 10 & $4165.30^{\text {cd }}$ & $4727.00^{\mathrm{c}}$ & $24.78^{\mathrm{ab}}$ & $2591.50^{\mathrm{cd}}$ & $1.35^{\mathrm{c}}$ & $550.53^{\mathrm{a}}$ & $34.81^{\mathrm{ab}}$ \\
\hline 13.3 & $4499.30^{\mathrm{bc}}$ & $5640.00^{\mathrm{bc}}$ & $25.72^{\mathrm{a}}$ & $3367.20^{\mathrm{b}}$ & $1.39^{\mathrm{bc}}$ & $553.25^{\mathrm{a}}$ & $35.37^{\mathrm{a}}$ \\
\hline 17.8 & $4629.20^{\mathrm{bc}}$ & $6869.30^{\mathrm{b}}$ & $24.38^{\mathrm{ab}}$ & $3861.50^{\mathrm{a}}$ & $1.34^{\mathrm{c}}$ & $546.65^{\mathrm{a}}$ & $34.98^{\mathrm{ab}}$ \\
\hline 20 & $5377.80^{\mathrm{a}}$ & $9003.20^{\mathrm{a}}$ & $24.05^{\mathrm{ab}}$ & $3272.80^{\mathrm{b}}$ & $1.44^{\mathrm{ab}}$ & $522.82^{\mathrm{a}}$ & $34.63^{\mathrm{ab}}$ \\
\hline 26.7 & $4858.20^{\mathrm{ab}}$ & $9334.30^{\mathrm{a}}$ & $23.68^{\mathrm{ab}}$ & $3072.80^{\mathrm{bc}}$ & $1.42^{\mathrm{b}}$ & $545.60^{\mathrm{a}}$ & $33.57^{\mathrm{ab}}$ \\
\hline 40 & $4211.20^{\mathrm{cd}}$ & $8948.30^{\mathrm{a}}$ & $23.28^{\mathrm{b}}$ & $2534.70^{\mathrm{d}}$ & $1.45^{\mathrm{ab}}$ & $488.43^{\mathrm{b}}$ & $33.67^{\mathrm{ab}}$ \\
\hline 80 & $3657.20^{\mathrm{d}}$ & $9324.50^{\mathrm{a}}$ & $23.38^{\mathrm{ab}}$ & $2205.30^{\mathrm{d}}$ & $1.51^{\mathrm{a}}$ & $480.33^{\mathrm{b}}$ & $32.75^{\mathrm{b}}$ \\
\hline SEM & 356.31 & 591.75 & 0.69 & 220.30 & 0.08 & 21.59 & 0.76 \\
\hline
\end{tabular}

Table 5: Interaction effects of cultivar and sowing density differences on yield parameters and crude protein (CP) contents of leaves and seeds of Canavaliaensiformis in 2012

\begin{tabular}{|c|c|c|c|c|c|c|c|c|}
\hline & & & Parameters & & & & & \\
\hline Cultivars & $\begin{array}{l}\text { Sowing } \\
\text { density }\end{array}$ & $\begin{array}{l}\text { LDMY } \\
(\mathrm{kg} / \mathrm{ha})\end{array}$ & $\begin{array}{l}\text { SDMY } \\
(\mathrm{kg} / \mathrm{ha})\end{array}$ & $\begin{array}{l}\text { LCP } \\
(\% \mathrm{DM})\end{array}$ & $\begin{array}{l}\text { SY } \\
(\mathrm{Kg} / \mathrm{ha})\end{array}$ & S:P & $\begin{array}{l}100 \text {-seed } \\
\text { weight }(\mathrm{g})\end{array}$ & $\begin{array}{l}\mathrm{SCP} \\
(\% \mathrm{DM})\end{array}$ \\
\hline \multirow[t]{7}{*}{ Black } & 10 & $4723.3^{\mathrm{cd}}$ & $5117.00^{\mathrm{e}}$ & 25.63 & $2381.30^{\mathrm{efg}}$ & $1.23^{\mathrm{e}}$ & $511.73^{\text {cde }}$ & $35.20^{\mathrm{abc}}$ \\
\hline & 13.3 & $5216.30^{\mathrm{bc}}$ & $6099.00^{\text {de }}$ & 26.30 & $2956.30^{\text {cde }}$ & $1.21^{\mathrm{e}}$ & $516.60^{\text {cde }}$ & $36.80^{\mathrm{a}}$ \\
\hline & 17.8 & $5782.00^{\mathrm{b}}$ & $8232.00^{\mathrm{bcd}}$ & 24.83 & $3361.00^{\mathrm{bcd}}$ & $1.19^{\mathrm{e}}$ & $493.13^{\text {de }}$ & $34.93^{\mathrm{abc}}$ \\
\hline & 20 & $6756.30^{\mathrm{a}}$ & $11455.00^{\mathrm{a}}$ & 23.90 & $2896.30^{\text {cdef }}$ & $1.25^{\mathrm{e}}$ & $485.80^{\mathrm{e}}$ & $36.33^{\mathrm{ab}}$ \\
\hline & 26.7 & $5379.70^{\mathrm{bc}}$ & $9903.00^{\mathrm{ab}}$ & 24.53 & $2852.30^{\mathrm{def}}$ & $1.27^{\mathrm{e}}$ & $512.03^{\text {cde }}$ & $33.53^{\mathrm{abc}}$ \\
\hline & 40 & $4366.30^{\mathrm{de}}$ & $9277.00^{\mathrm{ab}}$ & 23.73 & $2141.70^{\mathrm{fg}}$ & $1.25^{\mathrm{e}}$ & $438.27^{\mathrm{f}}$ & $34.30^{\mathrm{abc}}$ \\
\hline & 80 & $3706.00^{\mathrm{e}}$ & $9772.00^{\mathrm{ab}}$ & 23.73 & $1836.30^{\mathrm{g}}$ & $1.30^{\mathrm{e}}$ & $429.13^{\mathrm{f}}$ & $33.03^{b c}$ \\
\hline \multirow[t]{7}{*}{ White } & 10 & $3607.30^{\mathrm{e}}$ & $4337.00^{\mathrm{e}}$ & 23.93 & $2801.70^{\operatorname{def}}$ & $1.46^{\mathrm{d}}$ & $589.33^{\mathrm{a}}$ & $34.43^{\mathrm{abc}}$ \\
\hline & 13.3 & $3782.30^{\mathrm{e}}$ & $5181.00^{\mathrm{e}}$ & 25.13 & $3778.00^{\mathrm{ab}}$ & $1.56^{\mathrm{bcd}}$ & $589.90^{\mathrm{a}}$ & $33.93^{\mathrm{abc}}$ \\
\hline & 17.8 & $3476.30^{\mathrm{e}}$ & $5507.00^{\mathrm{e}}$ & 23.93 & $4362.00^{\mathrm{a}}$ & $1.49^{\mathrm{cd}}$ & $600.17^{\mathrm{a}}$ & $35.03^{\mathrm{abc}}$ \\
\hline & 20 & $3999.30 \mathrm{~d}^{\mathrm{e}}$ & $6551.00^{\text {bcd }}$ & 24.20 & $3649.30^{\mathrm{abc}}$ & $1.62^{\mathrm{ab}}$ & $559.83^{\mathrm{abc}}$ & $32.93^{\mathrm{bc}}$ \\
\hline & 26.7 & $4336.70 \mathrm{~d}^{\mathrm{e}}$ & $8766.00^{\mathrm{bc}}$ & 22.83 & $3293.30^{\mathrm{bcd}}$ & $1.58^{\mathrm{bc}}$ & $579.17^{\mathrm{ab}}$ & $33.60^{\mathrm{abc}}$ \\
\hline & 40 & $4056.00 \mathrm{~d}^{\mathrm{e}}$ & $8620.00^{\mathrm{bc}}$ & 22.83 & $2927.70^{\text {cde }}$ & $1.65^{\mathrm{ab}}$ & $538.60^{\mathrm{bcd}}$ & $33.03^{\mathrm{bc}}$ \\
\hline & 80 & $3608.30^{\mathrm{e}}$ & $8877.00^{\mathrm{b}}$ & 23.03 & $2574.30^{\text {defg }}$ & $1.71^{\mathrm{a}}$ & $531.53^{\text {bcde }}$ & $32.47^{\mathrm{c}}$ \\
\hline SEM & & 158.45 & 364.93 & 0.27 & 113.60 & 0.03 & 8.81 & 0.30 \\
\hline
\end{tabular}

$\mathrm{a}, \mathrm{b}, \mathrm{c}, \mathrm{d}:$ Means in same column with different superscripts are significantly $(\mathrm{p}<0.05)$ different

\section{Conclusion}

There were variations in the qualities of seed produced by the two cultivars of Jack bean. Both cultivars recorded similar leaf crude protein contents but the white-seeded cultivar had significantly lower seed crude protein content.

\section{References}

Adebowale, A. O. and Lawal 2004 Comparative study of the functional properties of Bambara groundnut (Voandzeia subterranean), jack bean (Canavaliaensiformis) and mucuna bean (Mucunapruriens) flours. Food Research International 
37(4):355-365 - May 2004.

DOI: 10.1016/j.foodres.2004.01.009

Akinlade, J. A., Farinu, G. O., Taiwo, A. A., Aderinola, D. A., Adebayo, T. A., Ojebiyi, O. O. and Olaniran, O. A. 2007. Agronomic and nutritive evaluation of Jack bean (Canaveliaensiformis) for fodder in the derived savannah zone of Nigeria. International Journal of Agricultural Research 2: 1059 1063

AOAC, 1995. Association of Official Analytical Chemists Official Methods of Analysis. 17 $7^{\text {th }}$ edition, Washinton DC, USA.

Arim, O. J., Waceke, J. W., Waudo, S. W. and Kimenju, J. W. 2006. Effects of Canaveliaensiformis and Mucunapuriens intercrops on PratylenchusZeae damage and yield of maize in subsistence agriculture. Plant and soil 284: 243 - 251 .

Bazill, Y. A. 1987. Evaluation of Tropical forage legumes under pinascaribeavarHondurensis in Coasta Rica, Turrialba. Agroforestry Systems 5: 97-108

Binuomote, R.T., Akinola, J.O., Akinlade, J.A. and Babayemi, O.J. 2009. Herbage yield and quality responses of plant components of Jack bean (Canavaliaensiformis)to planting density. Proceedings of the 14th Annual Conference of the Animal Science Association of Nigeria, LadokeAkintola University of Technology, Ogbomoso, Oyo State, Nigeria. 14-17 September, 2009.

Bogdan, A.V. 1977. Tropical pasture and fodder plants. Longman, London, UK, 475pp.

Chee, Y. K., Hacker, J. B., Ramirez, L. and Chen, C. P. 1992. Canavaliaensiformis (L.) DC.. Record from Proseabase. Mannetje,
L.'t and Jones, R.M. (Editors). PROSEA (Plant Resources of South-East Asia) Foundation, B o g o r, I n d o n e s i a. http://proseanet.org/prosea/eprosea detail.php

Fischler, M. and Wortmann, C. S. 1999. Green manures for maize-beans systems in Eastern Uganda: Agronomic Performance and Farmers' Perceptions. Agroforestry Systems 47, 123-138

Gomez, K.A and Gomez, A.A. 1984 Statistical Procedures for Agricultural Research. 2nd edn. New York: John Wiley and Sons.

Heuze, V. and Tran, G. 2015. Jack bean (Canavaliaensiformis) Feedipedia, a program by INRA, CIRAD, AFZ and FAO. H t t p : // w w w Feedipedia.org/node/827

Jimoh, W. A., Fagbenro,O. A. and Ad e parusi， E. O. 2010 . Digestibility coefficients of processed Jackbean meal Cannavaliaensiformis (L.) for Nile tilapia, Oreochromisniloticus (Linnaeus 1758) diets. International Journal of Fisheries and Aquaculture, Vol. 2(4): 102-107.

Liu, K. 1997. Soybeans, Chemistry, Technology and Utilization. Chapman and Hall, USA. Pg 532.

Morris, J. B. and Walker, J. T. 2002.Nontraditional legumes as potential soilamendments for nematode control. Journal of Nematology 34 (4) 358

NAS 1979. Tropical legumes' National Academy of Sciences. Washington DC. Pp 54-59.

Olowokudejo, J. O. Kadiri, A. B. and Travih, V. A. 2008 . A n Ethnobotanical survey of herbal markets and medicinal plants in Lagos State of Nigeria. Ethnoboatanical leaflets. An International Journal of 
Ethnobotanical Research. Southern Illinos University. Vol. 2008: Iss 1 A r t i c 1 e 116 . https://opensiuc.lib.siu.edu/eli/vol2 008/iss $1 / 116$

Pereira, B. F. F., Abreu, C. A.,Herpin, U. and Abreu, M. F. 2010. Phytoremediation of lead by jack beans on a RhodicHapludox amended with EDTA. Scientia Agricola 67 (3); $308-318$.
SAS 1988. User's Guide, Cary, North Carolina, USA: Statistical Analysis System Inc.

Udedibie, A. B. I. and Carlini, C. R.1998. Crack and cook: A simple and quick process for elimination of Concanaiahin A(Con A) from seeds. Animal Feed Science Technology 7: $179-184$

USDA2009.United States Department of Agriculture.

Received: $19^{\text {th }}$ September, 2021

Accepted: $10^{\text {th }}$ December, 2021 